Bundesgesundheitsbl 2014 · 57:22-26 DOI 10.1007/s00103-013-1868-1

Online publiziert: 20. Dezember 2013

๑) Springer-Verlag Berlin Heidelberg 2013

I. Schönfeld · K. Kraywinkel

Zentrum für Krebsregisterdaten, Robert Koch-Institut, Berlin

\title{
Krebsepidemiologische Daten im Internet
}

\section{Eine Übersicht über Informationsangebote im nationalen und europäischen Rahmen}

Die Suche nach zuverlässigen epidemiologischen Daten zu Krebserkrankungen im World Wide Web gestaltet sich nicht immer einfach. $\mathrm{Zu}$ verstreut sind die Angebote und die Quellen nicht immer eindeutig. Auch die Datengrundlagen unterscheiden sich teilweise erheblich, und die Zahlen untereinander sind nicht immer vergleichbar. An dieser Stelle soll eine kurze Übersicht gegeben werden über Informationsangebote nationaler und internationaler Organisationen, die verlässliche Daten für Deutschland und Europa bereitstellen ( $\bullet$ Infobox 1).

\section{Das Zentrum für Krebsregisterdaten (ZfKD): interaktive Abfrage mit Daten für Deutschland}

Zahlen zu Krebserkrankungen für Deutschland stellt das Zentrum für Krebsregisterdaten (ZfKD) im Robert Koch-Institut zur Verfügung (http:// www.krebsdaten.de/). Über ein interaktives Abfrageformular können Nutzerinnen und Nutzer ihre eigenen Tabellen zusammenstellen und Wertefilter anpas$\operatorname{sen}(\bullet$ Abb. 1).

Das ZfKD schätzt die Zahl jährlich neu aufgetretener Krebserkrankungen auf Grundlage der Daten aus den Landeskrebsregistern. Berechnet werden Erkrankungs-, Sterbe- und Überlebensraten sowie Prävalenzen für verschiedene Krebslokalisationen. Für die Altersstandardisierung kann zwischen Weltund Europastandard gewählt werden.
Die Daten zur Mortalität stammen aus der Todesursachenstatistik des Statistischen Bundesamtes. Die Abfrageergebnisse werden in Diagrammen visualisiert, es können Zeitverläufe oder Balkendiagramme dargestellt werden. Ein Export der Tabellen ist im csv- oder xls-Format möglich. Seit Kurzem veröffentlicht das ZfKD auch Inzidenzen (Neuerkrankungsraten) zu seltenen Tumoren in Deutschland, beispielsweise die des Mesothelioms. Ein Angebot, das sonst im internationalen Raum kaum zu finden ist: Daten zu seltenen Krebserkrankungen werden von vielen Registern nicht veröffentlicht.

Infobox 1 Auswahl von Webseiten nationaler und internationaler Organisationen, die verlässliche epidemiologische Daten zum Krebsgeschehen für Deutschland und Europa bereitstellen

Zentrum für Krebsregisterdaten: www.krebsdaten.de Gesellschaft der epidemiologischen Krebsregister in Deutschland e.V.: www.gekid.de

European Cancer Observatory: http://eco.iarc.fr/

GLOBOCAN-Projekt: http://globocan.iarc.fr/

Weitere relevante Seiten zu Krebserkrankungen, teilweise eher patientenorientiert: www.krebsinformationsdienst.de www.krebsgesellschaft.de www.krebshilfe.de 


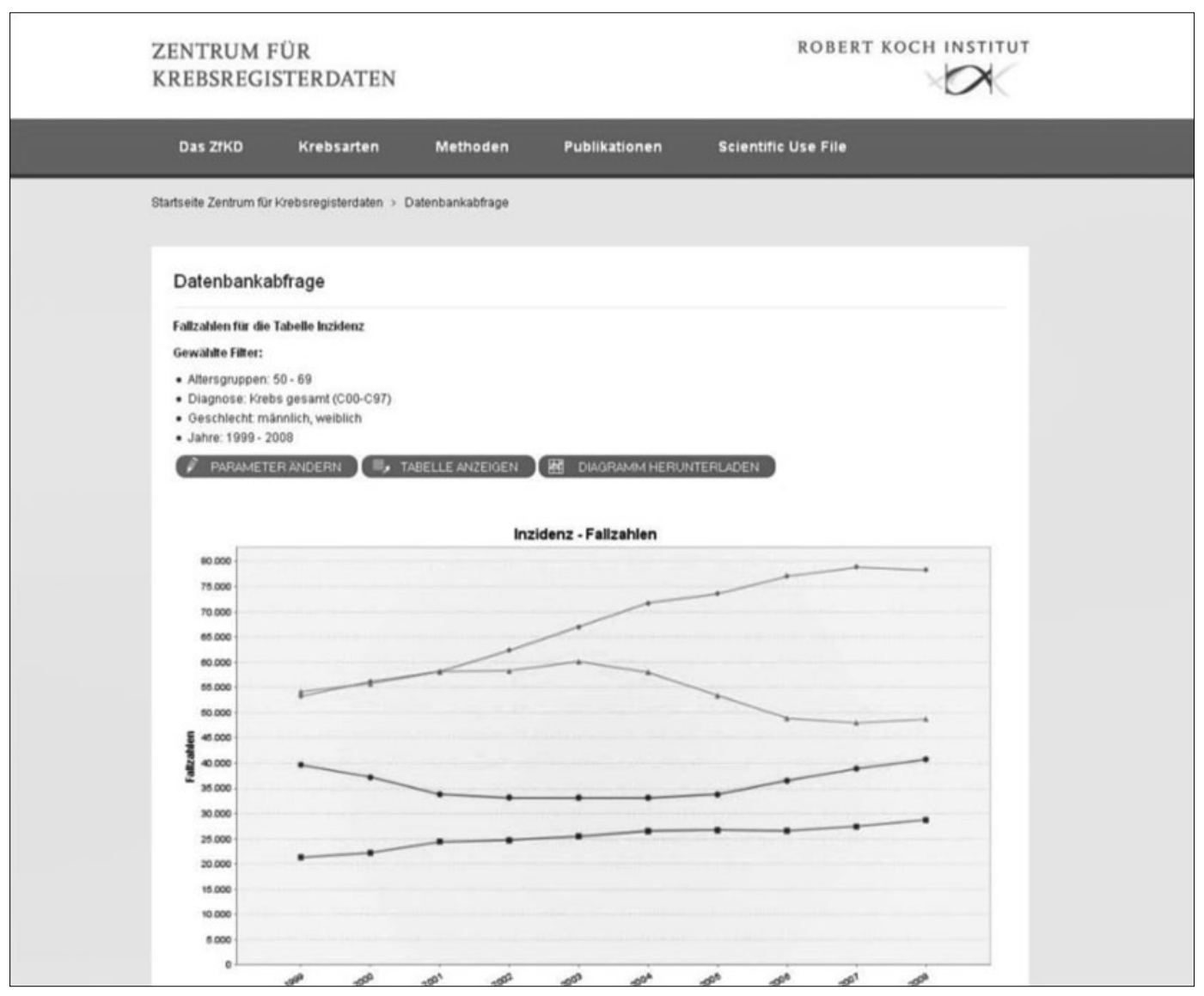

Abb. $1<$ Website des Zentrums für Krebsregisterdaten http://www. krebsdaten.de

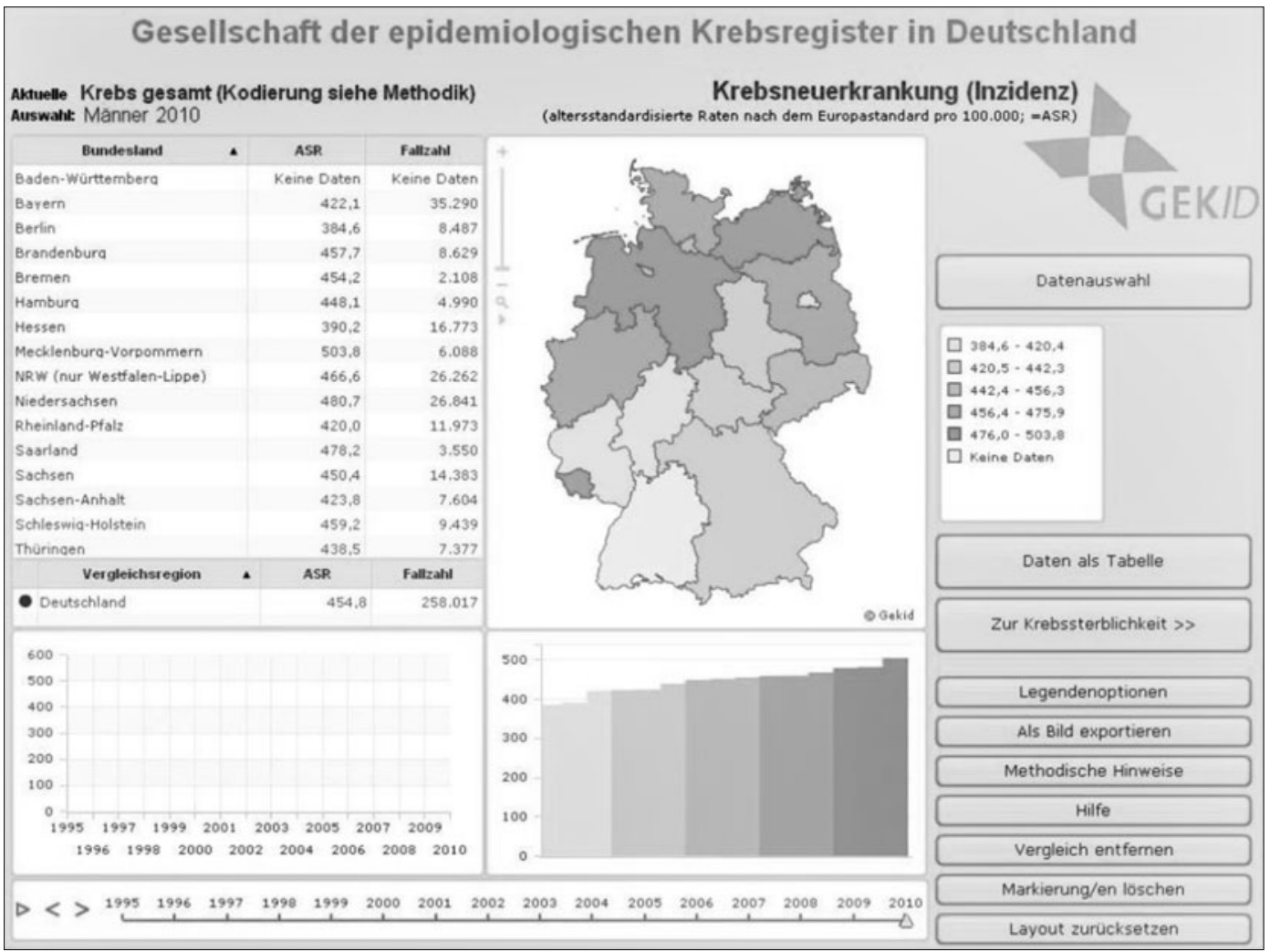

Abb. $2<$ Website der Gesellschaft der epidemiologischen Krebsregister in Deutschland e.V. http:// www.gekid.de 


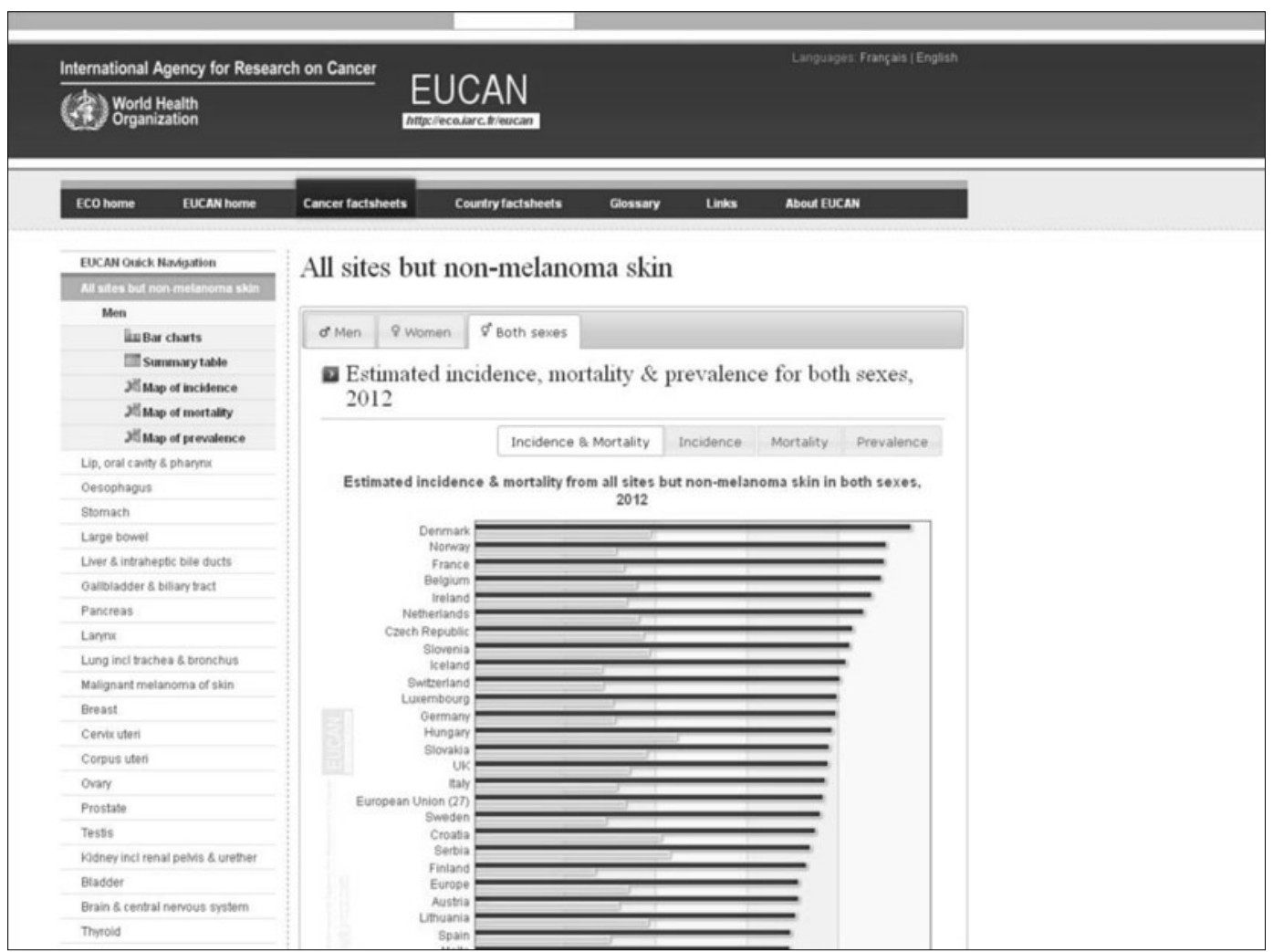

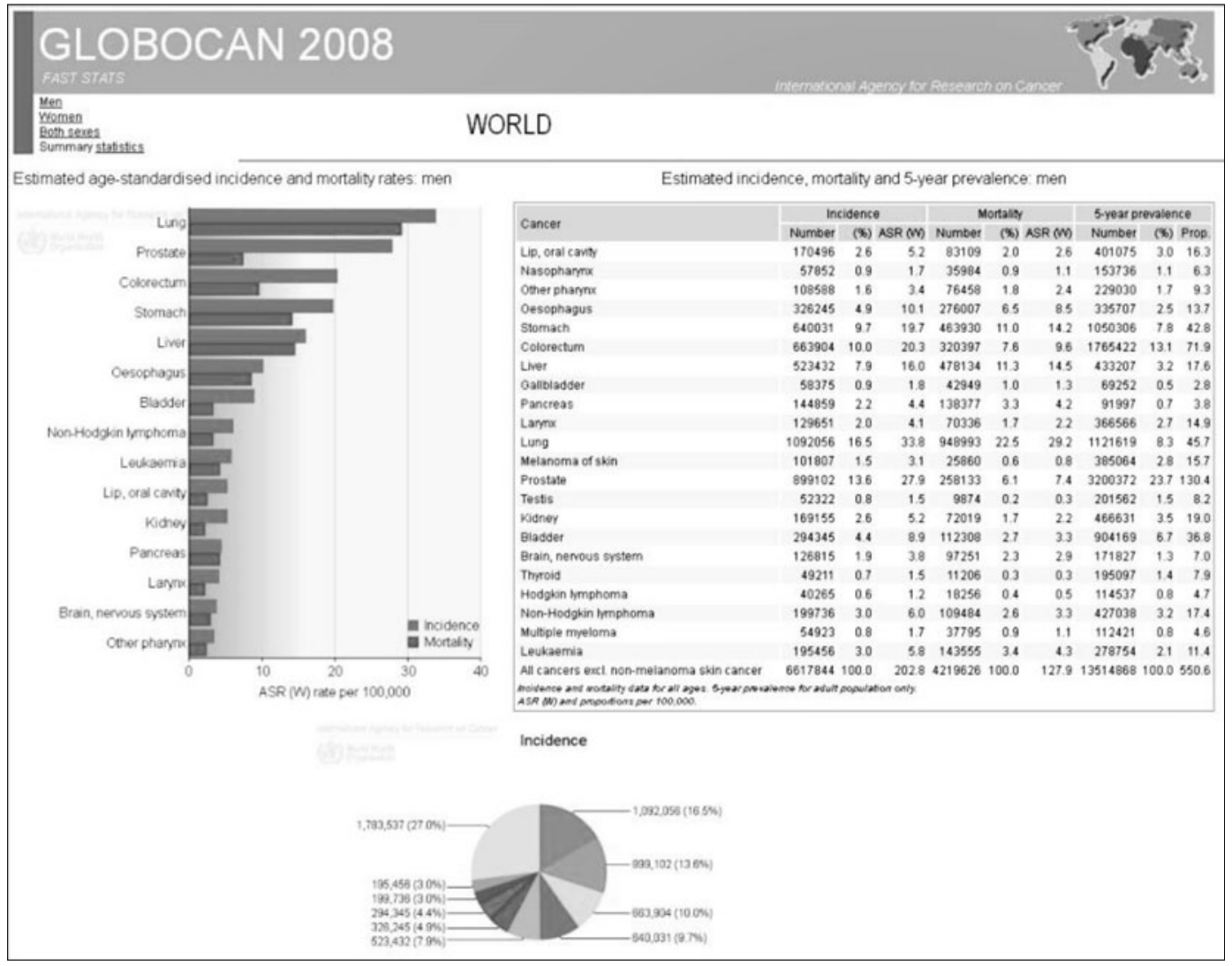

Abb. $3<$ Website der European Cancer Observatory http://eco.iarc.fr/
Abb. $4<$ Website des GLOBOCAN-Projektes http://globocan.iarc.fr/ 
Das Angebot des ZfKD geht jedoch über eine reine Datenbankabfrage hinaus. Das Formular ist eingebettet in allgemein verständliche Informationen zur Krebsepidemiologie. $\mathrm{Zu}$ den häufigen Krebsarten wird jeweils ein zusammenfassender Überblick über die Situation in Deutschland und zu möglichen Risikofaktoren gegeben. Die Berechnungsmethoden sind in einem eigenen Abschnitt erläutert.

Auf Antrag stellt das ZfKD den anonymisierten Datensatz als „Scientific Use File“ zur Verfügung. Die entsprechenden Antragsformulare finden sich auf der Website.

\section{Gesellschaft der epidemiologischen \\ Krebsregister in Deutschland e.V. (GEKID): kartografische Darstellung}

Ein weiteres krebsepidemiologisches Informationsportal für Deutschland stellt die Gesellschaft der epidemiologischen Krebsregister in Deutschland e.V. (GEKID; http://www.gekid.de/) mit ihrem Krebsatlas bereit ( $\bullet$ Abb. 2). In Karten wird hier die erfasste Inzidenz (Neuerkrankungsrate) aus den einzelnen Bundesländern dargestellt, je nach Beginn der Registrierung über unterschiedlich lange Zeitreihen. Eine über 10 ausgewählte Register gepoolte Inzidenzrate dient als Referenzwert. Die regionalen Unterschiede spiegeln jedoch teilweise eher Unterschiede in der Vollzähligkeit der Landesregister als tatsächliche Unterschiede in den Erkrankungsraten wider. Dargestellt werden absolute Fallzahlen und altersstandardisierte Inzidenz- und Sterberaten nach dem Europastandard. Der GEKID-Atlas setzt vor allem auf visuelle Darstellung. Das Layout kann farblich variiert und die Karten können in verschiedenen Formaten exportiert werden. Die in den Karten dargestellten Werte sind zusätzlich auch in Tabellen hinterlegt.

Auf der Website der GEKID findet sich unter anderem noch eine Übersicht zu den einzelnen Registern, die Links zu deren aktuellen Berichten und Internetauftritten beinhaltet. Die meisten Register stellen dort detaillierte, beispielswei- se auch altersspezifische Ergebnisse über eigene Datenbankabfragen zur Verfügung. Beim Deutschen Kinderkrebsregister in Mainz sind Tabellen zu Krebserkrankungen bei Kindern erhältlich (http://www.kinderkrebsregister.de).

\section{Das European Cancer Observatory (ECO): Quer- schnittsvergleich zwischen europäischen Ländern}

Das European Cancer Observatory (ECO; http://eco.iarc.fr/) ist ein Projekt, das die International Agency for Research on Cancer (IARC) in Zusammenarbeit mit dem European Network of Cancer Registries (ENCR) initiiert hat. Auf der Website sind Daten für 40 europäische Länder zusammengestellt, was sie besonders für Querschnittsvergleiche interessant macht (• Abb. 3).

Die Website ist in 3 Teile gegliedert. Während in einem Bereich namens „EUREG“ Daten aus den Krebsregistern Europas zusammengestellt sind, liefert ein zweites Segment („EUCAN“) eigene Schätzungen für die europäischen Länder. Der dritte Teil „EUROCIM“ soll künftig den Download von Datensätzen aus einzelnen Registern ermöglichen, war bei Redaktionsschluss jedoch noch nicht online verfügbar.

Für EUREG liefern etwa 100 epidemiologische Krebsregister ihre Daten zu Inzidenz, Mortalität und Überlebensraten an die IARC. Oft decken diese Register nicht ganze Länder, sondern nur einzelne Regionen ab. So sind für Deutschland Zahlen für die Bundesländer, nicht jedoch für Deutschland insgesamt erhältlich. Die Daten zur Krebsmortalität in EU-Staaten sind nicht so aktuell wie die auf der Website von EUROSTAT, dem Statistischen Amt der Europäischen Union, das in diesem Punkt sehr detaillierte und aktuelle Tabellen bereithält.

Die nationalen Schätzungen für EUCAN beruhen auf den Daten der jeweils aktuellen Ausgabe von "Cancer Incidence in Five Continents" die die IARC alle 5 Jahre herausgibt. Die Zahlen sind damit immer Prognosen bzw. Hochrechnungen aus älteren Daten und beziehen sich aktuell auf das Jahr 2012. Dargestellt
Bundesgesundheitsbl 2014 · 57:22-26

DOI 10.1007/s00103-013-1868-1

๑) Springer-Verlag Berlin Heidelberg 2013

\section{Schönfeld $\cdot$ K. Kraywinkel \\ Krebsepidemiologische \\ Daten im Internet. Eine Übersicht über Informations- angebote im nationalen und europäischen Rahmen}

\section{Zusammenfassung}

Die Suche nach krebsepidemiologischen Daten im World Wide Web ist nicht immer einfach. Die Angebote sind verstreut und die Quellen oft nicht eindeutig. Der Artikel gibt eine kurze Übersicht über die wichtigsten Informationsangebote, die verlässliche Daten für Deutschland und Europa bereitstellen. Vorgestellt werden die Websites des Zentrums für Krebsregisterdaten (ZfKD), der Gesellschaft der epidemiologischen Krebsregister in Deutschland e.V. (GEKID) und 2 Portale der International Agency for Research on Cancer (IARC). In Kombination ergeben diese ein umfassendes Bild über die Verteilung von Krebserkrankungen in Deutschland und Europa.

Schlüsselwörter

Krebs · Statistik $\cdot$ Krebsdaten · Deutschland . Europa

Epidemiological cancer data online. An overview of information service in Germany and Europe

\section{Abstract}

Finding reliable data about cancer epidemiology on the World Wide Web is not an easy task. Information is often scattered, and sources are not always clear. This article gives a short overview of the most important websites that provide reliable data for Germany and Europe. Four internet sites are presented: The German Centre for Cancer Registry Data (ZfKD), the Association of PopulationBased Cancer Registries in Germany (GEKID), and two different websites created by the International Agency for Research on Cancer (IARC). In combination, they provide comprehensive information about the distribution of cancer in Germany and Europe.

Keywords

Cancer - Statistics - Cancer data - Germany . Europe

werden Inzidenz, Mortalität und Prävalenz für 40 europäische Länder. Zeitliche Verläufe werden nicht angegeben. 


\section{Leitthema}

Es ist möglich, sich auf der ECOWebsite zu registrieren. Eingeloggten Nutzerinnen und Nutzern stehen weitere Funktionalitäten bereit, beispielsweise lassen sich Suchkriterien und LayoutEinstellungen speichern.

\section{Das GLOBOCAN-Projekt: weltweite Schätzungen}

Für 184 Länder schätzen die Wissenschaftlerinnen und Wissenschaftler des GLOBOCAN Projects (http://globocan. iarc.fr/) der IARC Inzidenz, Mortalität und Prävalenz der häufigsten Krebserkrankungen (• Abb.4). Die Datengrundlagen unterscheiden sich jedoch von Land zu Land zum Teil erheblich. In einigen Fällen werden für die Berechnung Daten aus Nachbarländern herangezogen und extrapoliert. Eine wichtige Datenquelle ist auch hierfür „Cancer Incidence in Five Continents".

Im Vordergrund steht die Darstellung, wie sich die Krankheitslast durch Krebs weltweit verteilt. Die Kategorien umfassen - neben einzelnen Ländern auch größere Regionen wie Kontinente oder Teilkontinente. Auch ein Vergleich von entwickelten und weniger entwickelten Ländern ist möglich. Der Altersstandardisierung liegt der Weltstandard zugrunde.

\section{Fazit}

Einen Anspruch auf Vollständigkeit erhebt diese Zusammenstellung selbstverständlich nicht. Zweifellos gibt es auch noch andere Quellen, die hier nicht explizit genannt wurden. Oft sind dort jedoch nur einzelne Regionen oder bestimmte Parameter abrufbar. Die vorgestellten Websites bieten einen guten Überblick über nationale und internationale krebsepidemiologische Daten. Da die Abfrageformulare oftmals vielfältig und die Möglichkeiten komplex sind, erfordert es in der Regel etwas Geduld, sich mit den Oberflächen vertraut zu machen. Ist man bereit, diese aufzubringen, erhält man jedoch umfassende Informationen zu verschiedenen Krebsarten und Parametern.

\section{Korrespondenzadresse}

Dipl.-Biol. I. Schönfeld

Zentrum für Krebsregisterdaten, Robert Koch-Institut

General-Pape-Str. 62-66, 12101 Berlin

schoenfeldl@rki.de

\section{Einhaltung ethischer Richtlinien}

Interessenkonflikt. I. Schönfeld und K. Kraywinkel geben an, dass kein Interessenkonflikt besteht.

Dieser Beitrag beinhaltet keine Studien an Menschen oder Tieren. 